
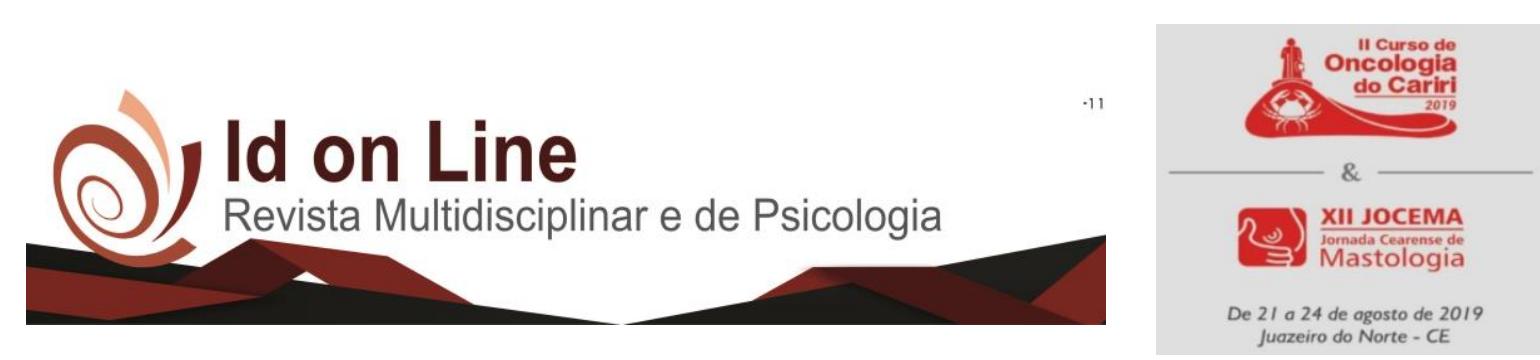

DOI: 10.14295/idonline.v13i46.1997

Resumo

\title{
USO DE DERIVADOS DO ÁCIDO ACETILSALICÍLICO PARA REDUÇÃO DO RISCO DE DESENVOLVIMENTO DE NEOPLASIAS PANCREÁTICAS
}

\author{
PINHO, Jonas Lima ${ }^{\mathbf{1}}$; RAMIREZ, Fabian Danilo Unigarro'; FILGUEIRAS, Ana Clara Tavares ${ }^{\mathbf{1}}$; \\ DOMINGOS, Vinicius Gonçalves ${ }^{1}$; LIMA, Iri Sandro Pampolha ${ }^{2}$
}

Introdução: O câncer de pâncreas é uma doença que, apesar dos tratamentos que hoje existem, ainda continua com baixas taxas de sobrevida. Têm sido testadas várias opções de tratamento relativamente seguras e baratas que obtiveram sucesso em alguns modelos pré-clínicos. Estudos epidemiológicos esclareceram as possíveis associações entre o uso regular de aspirina e cânceres. A terapia com aspirina e com fármacos derivados do mesmo, tem demonstrado prevenir o câncer de pâncreas e pode ser útil na redução da recorrência de neoplasias pancreáticas na população. Objetivos: Analisar a eficácia, benefícios e toxicidade que o uso crônico de aspirina e seus derivados têm na diminuição do risco de desenvolvimento de neoplasias pancreáticas. Metodologia: O presente trabalho consiste numa revisão sistemática norteada pela metodologia PRISMA, abordando estudos primários publicados na base de dados "Scopus (Elsevier)" entre 2016 e 2019 com os descritores: "cancer", "pancreas" e "aspirin". Os critérios de inclusão foram: artigos publicados nos últimos 4 anos, em inglês e espanhol, que associassem o uso de aspirina à redução do risco de desenvolver neoplasias pancreáticas. Excluíram-se comentários, revisões editoriais e cartas ao editor. Resultados: 23 estudos foram rastreados. Após leitura dos títulos e resumos e, posterior aplicação dos critérios de inclusão/exclusão, obtivemos uma amostra de 6 artigos. Estes estudos pontuam que o uso regular de aspirina parece reduzir o risco de câncer pancreático em quase metade e que a chance de desenvolver neoplasias pancreáticas diminuiu $8 \%$ por cada ano acumulado de uso de AAS. Além disso, pesquisas identificaram que a associação de AAS com Atorvastatina diminui o crescimento tumoral e aumenta a eficácia de quimioterápicos como a Gemcitabina. No entanto, é sabido que o uso contínuo de AAS ou outros AINES predispõe nos indivíduos complicações hemorrágicas e lesões na mucosa gástrica. Nesse sentido, foram desenvolvidos fármacos derivados da aspirina, como a fosfo-aspirina, que, em modelos préclínicos usando ratos, pareceu ser mais segura, não mostrando sinais de toxicidade durante o tratamento. Conclusão: É necessário que novas pesquisas sejam desenvolvidas com o fito de produzir novos tratamentos mais eficazes e menos invasivos que os atualmente disponíveis, garantindo, assim, uma maior taxa de sobrevida e uma diminuição do desenvolvimento deste tipo de câncer.

Palavras-chave: câncer, pâncreas, aspirina, fosfo-aspirina, ácido acetilsalicílico.

\footnotetext{
${ }^{1}$ Acadêmicos do curso de Medicina da Universidade Federal do Cariri (UFCA). jonaslpinho@gmail.com, fabiunir@hotmail.es, anaclaratfilgueiras@gmail.com, vinidomingos1@gmail.com;

${ }^{2}$ Professor Adjunto do curso de Medicina da Universidade Federal do Cariri (UFCA). irisandro@ gmail.com.
} 


\section{Referências}

BIGELSEN, Stephen. Evidence-based complementary treatment of pancreatic cancer: a review of adjunct therapies including paricalcitol, hydroxychloroquine, intravenous vitamin $\mathrm{C}$, statins, metformin, curcumin, and aspirin. Cancer Management And Research, [s.l.], v. 10, p.20032018, jul. 2018. Dove Medical Press Ltd.

LIU, Qiaofei et al. Atorvastatin (Lipitor) attenuates the effects of aspirin on pancreatic cancerogenesis and the chemotherapeutic efficacy of gemcitabine on pancreatic cancer by promoting M2 polarized tumor associated macrophages. Journal Of Experimental \& Clinical Cancer Research, [s.1.], v. 35, n. 1, p.33-49, 16 fev. 2016. Springer Nature.

MATTHEOLABAKIS, G. et al. Phospho-Aspirin (MDC-22) Prevents Pancreatic Carcinogenesis in Mice. Cancer Prevention Research, [s.1.], v. 9, n. 7, p.624-634, 2 maio 2016. American Association for Cancer Research (AACR).

QIAO, Yan et al. Associations between aspirin use and the risk of cancers: a meta-analysis of observational studies. Bmc Cancer, [s.1.], v. 18, n. 1, p.18-288, 13 mar. 2018. Springer Nature.

RISCH, Harvey A. et al. Aspirin Use and Reduced Risk of Pancreatic Cancer. Cancer Epidemiology Biomarkers \& Prevention, [s.1.], v. 26, n. 1, p.68-74, 20 dez. 2016. American Association for Cancer Research (AACR).

TAKASAKI, Yusuke et al. Effect of low-dose aspirin use on pancreatic cancer development and morphological changes on imaging in IPMN: A long-term cohort study. United European Gastroenterology Journal, [s.1.], v. 5, n. 7, p.1030-1036, 5 fev. 2017. SAGE Publications. 